\title{
Aplicação das Opções Compostas na Avaliação da Opção de Venda Americana
}

\author{
José Ferreira Marinho Junior* \\ Mauro Antonio Rincon**
}

Resumo

Neste artigo, um método numérico é desenvolvido para se encontrar o valor de uma opção de venda americana, baseado na solução de Black e Scholes (1973) para opções européias e na extrapolação de Richardson, que calcula o limite de uma sequência de opções, cujos intervalos de tempo tendem a zero. No início da década de 1970, Black e Scholes (1973) e Merton (1973) desenvolveram uma equação diferencial parcial, cuja solução determina o valor de uma opção européia. A condição de fronteira irá determinar o tipo de opção (compra ou venda). Valores para a opção de venda americana são calculados, tabelados e comparados com os métodos da integração numérica e da aproximação binomial.

Palavras-chave: opções compostas; extrapolação de Richardson, opção americana; método de Newton-Raphson.

Códigos JEL: C02; C15; C63.

\section{Abstract}

In this article, a numerical method is developed to determine the value of a put, based in the solution of Black and Scholes (1973) for European option and on Richardson extrapolation, that calculates the limit of an options sequence, whose time intervals tend to zero. In the beginning of the 70's, Black and Scholes (1973) and Merton (1973) they had developed partial differential equation, whose solution it determines the value of an European option. The boundary condition will go to determine the type of option (purchase or sale). Values for the put are calculated, priced and compared with methods of the numerical integration and the binomial approach.

Keywords: compound options; Richardson extrapolation; american put; Newton-Raphson method.

\section{Introdução}

Merton (1973) mostrou que opções de venda americana são mais difíceis de calcular o valor do que opções de venda européia porque na opção americana existe uma probabilidade positiva de ser exercida prematuramente. Black e Scholes (1973) derivaram uma equação diferencial que deve ser satisfeita pelo preço de qualquer derivativo dependente de uma ação sem dividendos. A solução desta equação diferencial fornece o preço de uma opção européia.

$$
\frac{\partial P}{\partial S} S r+\frac{\partial P}{\partial t}+\frac{1}{2} \frac{\partial^{2} P}{\partial S^{2}} \sigma^{2} S^{2}=P r
$$

Submetido em Novembro de 2005. Revisado em Dezembro de 2006.

*Instituto de Matemática, Universidade Federal do Rio de Janeiro, Caixa Postal 68530, Rio de Janeiro 21945-970, Brazil. E-mail: josefmj@posgrad.nce.ufrj.br

**E-mail: rincon@dcc.ufrj.br 
onde $P$ é o valor da opção, $S$ é preço do ativo-objeto, $\sigma$ é a volatilidade, $r$ é a taxa de juros livre de risco e $t$ é o tempo para o vencimento.

A equação (1) é a equação diferencial de Black e Scholes (1973), cuja solução depende das condições de fronteira utilizadas.

Para uma opção de venda européia, a principal condição de fronteira é:

$$
P=\max (S-X, 0)
$$

onde $X$ é o preço de exercício.

A solução para a condição (2), demonstrada por Black e Scholes (1973), é dada por,

$$
P=X e^{-r t} N\left(-d_{2}\right)-S N\left(-d_{1}\right)
$$

onde

$$
d_{1}=\frac{\ln (S / X)+\left(r+\sigma^{2} / 2\right) t}{\sigma \sqrt{t}}, \quad d_{2}=d_{1}-\sigma \sqrt{t}
$$

e $N(x)$ é a função de distribuição de probabilidade acumulada para uma variável que é distribuída normalmente, com média zero e desvio padrão um, ou seja, é a probabilidade da variável ser menor que $x$ e dado por,

$$
N(x)=\frac{1}{\sqrt{2 \pi}} \int_{x}^{\infty} e^{-y^{2} / 2} d y
$$

\section{Formulação}

Para solucionar o problema de avaliação da opção de venda americana, seja $P\left(S_{0}, \sigma^{2}, r, T, X\right)$ o preço da opção de venda americana.

Segue-se as hipóteses utilizadas por Black e Scholes (1973), considerando o mercado perfeito, $r$ e $\sigma$ constantes e movimento browniano geométrico para o ativo-objeto. O tempo atual é definido como zero e o processo estocástico para as mudanças no preço do ativo-objeto é:

$$
\frac{d S}{S}=\mu d t+\sigma d z
$$

onde $\mu$ é o retorno esperado do ativo-objeto e $d z$ é o processo de Wiener. Como apenas o preço do ativo e o tempo são considerados variáveis e o preço do ativo é estocástico, então as mudanças no preço da opção de venda americana podem ser caracterizadas pelo Lema de Itô. Do mesmo modo utilizado por Black e Scholes (1973), constrói-se uma carteira protegida com uma posição vendida no derivativo e outra comprada em $\partial P / \partial S$ ativos.

Seguindo todo o processo utilizado em Black e Scholes (1973), o preço da opção de venda americana é descrito pela equação diferencial parcial do tipo parabólico, 


$$
\frac{\partial P}{\partial t}=r P-r S \frac{\partial P}{\partial S}-\frac{1}{2} \sigma^{2} S^{2} \frac{\partial^{2} P}{\partial S^{2}}
$$

sob a seguinte condição final:

$$
P(S, T) \geq \max (X-S, 0), \quad \forall T \geq 0
$$

Como a opção de venda americana pode ser exercida a qualquer instante até o vencimento, o titular sempre irá se perguntar se um determinado instante é ótimo. Num primeiro instante ele verifica o valor do ativo-objeto e busca no futuro um valor menor. Caso encontre, não irá exercer a opção e continuar verificando até o vencimento.

Pode-se dizer então que esta situação é equivalente a uma sequência infinita de opções sobre opções, ou opções compostas.

Existem quatro tipos de opções compostas: uma opção de compra sobre uma opção de compra, uma opção de compra sobre uma opção de venda, uma opção de venda sobre uma opção de compra e uma opção de venda sobre uma opção de venda. As opções compostas possuem dois preços de exercício e duas datas de exercício (ou dois tempos para o vencimento). Considerando o movimento browniano geométrico para o preço do ativo, o valor de uma opção de compra sobre uma opção de compra é avaliado analiticamente em termos da distribuição normal bivariada. O valor desta opção composta, sem dividendos, é dado, como em (5) por:

$$
\begin{aligned}
P & =S N_{2}\left(d_{1},-k_{1}\right) \\
& \left.-\sqrt{T_{1} / T_{2}}\right)-X_{2} e^{-r T_{2}} N_{2}\left(d_{2},-k_{2}\right. \\
& \left.-\sqrt{T_{1} / T_{2}}\right)+e^{-r T_{1}} X_{1} N\left(d_{2}\right)
\end{aligned}
$$

onde, $P$ é o valor desta opção composta, $X_{1}$ é o preço de exercício no instante $T_{1}$, $X_{2}$ é o preço de exercício no instante $T_{2}$,

$$
\begin{array}{ll}
d_{1}=\frac{\ln (S / \bar{S})+\left(r+\sigma^{2} / 2\right) T_{1}}{\sigma \sqrt{T_{1}}}, & d_{2}=d_{1}-\sigma \sqrt{T_{1}} \\
k_{1}=\frac{\ln \left(S / X_{2}\right)+\left(r+\sigma^{2} / 2\right) T_{2}}{\sigma \sqrt{T_{2}}}, & k_{2}=k_{1}-\sigma \sqrt{T_{2}}
\end{array}
$$

Sendo $N_{2}$ a função de distribuição normal bivariada acumulada. A variável $\bar{S}$ é o preço do ativo no instante $T_{1}$, pelo qual o preço da opção se iguala $X$. Se o preço do ativo estiver abaixo de $\bar{S}$ em $T_{1}$, a opção será exercida nesse instante. $\quad N_{2}\left(d_{1},-k_{1} ;-\sqrt{T_{1} / T_{2}}\right)$ representa a probabilidade acumulada de uma distribuição normal bivariada padronizada do preço do ativo-objeto, no primeiro instante, estar acima de $\bar{S}$ e, no segundo instante, ser menor que o preço de exercício, $X$, quando o coeficiente de correlação entre as variáveis é $\left(-\sqrt{T_{1} / T_{2}}\right)$. 
Como a opção de venda americana pode ser exercida a qualquer instante até o vencimento, ela é equivalente a uma sequência infinita de opções compostas.

Para encontrar a solução do problema considera-se que cada decisão de exercício é um evento discreto. Então a fórmula deverá ser uma solução em tempo contínuo para a equação (5) sujeita a condição de fronteira (6) aplicada a um número infinito de eventos discretos.

Em cada instante, a opção será exercida se:

- Não foi exercida anteriormente;

- O pagamento pelo exercício for maior ou igual a opção caso ela não seja exercida.

Portanto, em cada instante discreto, no qual há possibilidade de exercício (chamado de ponto de exercício), é calculado um valor para o ativo. Este valor será chamado de preço crítico $(\bar{S})$ e determinará se haverá ou não exercício naquele instante. A opção será exercida se num determinado instante, o preço do ativo for menor que o preço crítico deste instante, dado que o preço do ativo sempre foi maior que o preço crítico nos instantes anteriores, pois não houve exercício. $\mathrm{O}$ preço crítico do ativo é independente do preço atual do ativo e é determinado a partir da fronteira livre, dada pela equação (6), sempre que

$$
X-\bar{S}=P(\bar{S}, T)
$$

para algum $S=\bar{S}$ e qualquer $T$.

Esquematicamente, tem-se:

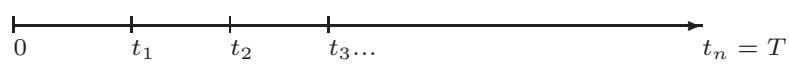

Em $t_{i}$ observa-se o seguinte:

$$
\begin{aligned}
& S_{t_{i}}=\text { valor do ativo em } t_{i} \\
& \bar{S}_{t_{i}}=\text { preço crítico em } t_{i} ; \bar{S}_{t_{i}} \in\left(t_{i}, T\right]
\end{aligned}
$$

A opção será exercida no instante $t_{i}$, se $S_{t_{i}}<\bar{S}_{t_{i}}$ e $S_{t_{j}}>\bar{S}_{t_{j}}, \forall j<i$.

A discretização utilizada será uma sequência em que cada conjunto de oportunidades inclui a anterior, isto é, a oportunidade da opção com 1 ponto de exercício deve estar contida na opção com 2 pontos de exercício, cujas oportunidades devem estar contidas na opção seguinte. A próxima opção que irá conter todas as oportunidades da opção com 2 pontos de exercício é a opção com 4 pontos de exercício. Portanto, deve-se utilizar passos geométricos no tempo. Utilizar passos geométricos significa discretizar o tempo em $1,2,4,8, \ldots$ intervalos de tempo iguais.

Seja $P_{1}$ a opção que só tenha um ponto de exercício e suponha que este ponto seja no vencimento. Nesse caso, $P_{1}$ é equivalente a opção européia e o preço crítico para o instante $T$ é o próprio preço de exercício. 
Portanto, por Black e Scholes (1973):

$$
P_{1}=X e^{-r T} N\left(-d_{2}\left(S_{0}, X, T\right)\right)-S_{0} N\left(-d_{1}\left(S_{0}, X, T\right)\right)
$$

onde:

$$
d_{1}\left(S, \overline{S_{t}}, t\right)=\frac{\ln \left(S / \overline{S_{t}}\right)+\left(r+(1 / 2) \sigma^{2}\right) t}{\sigma \sqrt{t}} \quad \text { e } \quad d_{2}\left(S, \overline{S_{t}}, t\right)=d_{1}-\sigma \sqrt{t}
$$

Antes de calcular o valor da opção com dois pontos de exercício, considera-se

$$
\bar{S} \equiv\left\{\bar{S}_{t}, \bar{S}_{t} \geq 0, \quad t \in[0, T]\right\}
$$

o conjunto dos preços críticos que denota uma fronteira onde o exercício antecipado, ou seja, antes do vencimento é ótimo. A função $P\left(S_{t}, t\right) \in C^{1,1} \mathrm{em}$ $[\bar{S}, \infty] \times[0, T]$ e é de classe $C^{2,1}$ em $(\bar{S}, \infty] \times[0, T]$. Como a condição de fronteira da opção de venda americana no vencimento é $P\left(S_{T}, T\right)=\max (X-S, 0)$ então:

$$
\begin{gathered}
\lim _{S_{t} \rightarrow \infty} P\left(S_{t}, t\right)=0 \\
\lim _{S_{t} \rightarrow \bar{S}_{t}} P\left(S_{t}, t\right)=X-\bar{S}_{t} \\
\lim _{S_{t} \rightarrow \bar{S}_{t}} \frac{\partial P\left(S_{t}, t\right)}{\partial S_{t}}=-1
\end{gathered}
$$

Seguindo a direção dada por Carr et alii (1992), a expressão para a opção de venda americana sem dividendos é:

$$
P=p_{0}+r X \int_{0}^{T} e^{-r t} N\left(\frac{\ln \left(S_{t} / S_{0}\right)-\rho_{2} t}{\sigma \sqrt{t}}\right) d t
$$

onde $p_{0}$ é o valor da opção de venda européia e $N(x)$ é a função de distribuição de probabilidade acumulada.

Esta equação pode ser interpretada da seguinte maneira:

- A primeira parcela, valor da opção européia, é o valor do pagamento garantido da opção de venda, ou seja, no mínimo a opção de venda americana vale o mesmo que a européia no caso do vencimento ter sido o momento ótimo para o exercício;

- A segunda parcela é o valor presente dos benefícios do exercício antecipado da opção, ganhos através dos juros recebidos com o exercício. 
Seja, agora, $P_{2}$ a opção que só tenha dois pontos de exercício e suponha que estes pontos sejam no vencimento e na metade do tempo para o vencimento.

No instante $T / 2$ não existe a probabilidade da opção já ter sido exercida anteriormente então calcula-se a probabilidade da opção ser exercida neste instante. No vencimento, $2^{\circ}$ ponto de exercício, deve-se calcular a probabilidade da opção ser exercida neste ponto, dado que para chegar até este instante ela não foi exercida no ponto de exercício anterior. Daí, temos:

$$
P_{2}=\widehat{P}_{1}+\frac{r X T}{2} e^{-r T / 2} N_{1}\left(-d_{2}\left(S_{0}, \bar{S}_{T / 2}, T / 2\right)\right)
$$

onde $\widehat{P}_{1}$ é a probabilidade da opção ser exercida no primeiro ponto de exercício, ou seja, em $t=T / 2$, calculada por Black e Scholes (1973), para um intervalo de tempo $\Delta t=T / 2$ com o preço de crítico deste instante denotado por $\bar{S}_{T / 2}$.

Seja, agora, $P_{4}$ a opção que tenha quatro pontos de exercício e suponha que estes pontos sejam em 1/4 do tempo, na metade do tempo, em 3/4 do tempo e no vencimento. No instante $T / 4$ não existe a probabilidade da opção já ter sido exercida anteriormente então calcula-se a probabilidade da opção ser exercida neste instante. No $2^{\circ}$ ponto de exercício, instante $T / 2$, deve-se calcular a probabilidade da opção ser exercida neste ponto, dado que para chegar até este instante ela não foi exercida no ponto de exercício anterior. No $3^{\circ}$ ponto de exercício, instante $3 T / 4$, deve-se calcular a probabilidade da opção ser exercida neste ponto, dado que para chegar até este instante ela não foi exercida nos dois pontos de exercício anteriores. E no vencimento, $4^{\circ}$ ponto de exercício, deve-se calcular a probabilidade da opção ser exercida neste ponto, dado que para chegar até este instante ela não foi exercida nos três pontos de exercício anteriores. Daí, temos:

$$
\begin{aligned}
P_{4} & =\widehat{P}_{1}+\frac{r X T}{4}\left[e^{-r T / 4} N_{1}\left(-d_{2}\left(S_{0}, \bar{S}_{T / 4}, T / 4\right)\right)\right. \\
& +e^{-r T / 2} N_{1}\left(-d_{2}\left(S_{0}, \bar{S}_{T / 2}, T / 2\right)\right) \\
& \left.+e^{-r 3 T / 4} N_{1}\left(-d_{2}\left(S_{0}, \bar{S}_{3 T / 4}, 3 T / 4\right)\right)\right]
\end{aligned}
$$

Os preços críticos podem ser determinados em qualquer instante para toda data futura antes do vencimento. No vencimento o preço crítico é o próprio preço de exercício, pois é o último instante em que pode haver exercício. Portanto para calcular o valor de $P_{1}$ (que somente tem um ponto de exercício), basta usar diretamente a fórmula de Black e Scholes (1973). No valor de $P_{2}$ é necessário calcular o preço crítico do instante $T / 2$ e isto é feito utilizando-se a função $P_{1}$ e considerando que $\bar{S}_{T / 2}$ seria o $S_{0}$ da função $P_{1}$ com intervalo de tempo $\Delta t=T / 2$. Assim, $\bar{S}_{T / 2}$ é solução da equação:

$$
P_{1}(S, X, T / 2)=X-S
$$

Seguindo esta formulação, calcula-se os preços críticos $\bar{S}_{T / 4}, \bar{S}_{T / 2}$ e $\bar{S}_{3 T / 4}$ necessários para calcular o valor de $P_{4}$. 
Para encontrar os preços críticos utiliza-se o método de Newton-Raphson onde $f(S)=X-S-P$ de tal forma que:

$$
S_{i+1}=S_{i}-\frac{f\left(S_{i}\right)}{f^{\prime}\left(S_{i}\right)}, \quad i=0,1,2, \cdots
$$

e a aproximação inicial $S_{0}$ será o valor de $S_{0}$, ou seja, o preço inicial do ativo.

Conhecidos os preços críticos, o objetivo agora é encontrar o valor de $P$. O valor da opção de venda americana será o limite da sequência $\left\{P_{n}, \quad n \in \mathbf{N}\right\}$. Existem várias técnicas para calcular este tipo de limite. A técnica aqui utilizada será a extrapolação de Richardson.

A extrapolação de Richardson permite a determinação do valor limitante de alguma sequência conforme o "comprimento do passo" $h$ se aproxima de zero. Neste problema, o comprimento do passo é o intervalo de tempo entre os pontos de exercício. Esta extrapolação leva a uma equação polinomial que pode ser usada para determinar os valores da opção de venda americana.

Para aplicar esta extrapolação no problema de apreçamento da opção de venda americana, considera-se $P_{1}=F(h)$ a função com passo de tamanho $h$ com a seguinte forma:

$$
F(h)=a_{0}+a_{1} h+a_{2} h^{2}+O\left(h^{3}\right)
$$

O objetivo é determinar o valor de $a_{0}$, pois $\lim _{h \rightarrow 0} F(h)=a_{0}$.

Desta forma, pode-se escrever $P_{2}=F\left(\frac{h}{2}\right)$ tal que:

$$
F\left(\frac{h}{2}\right)=a_{0}+a_{1}\left(\frac{h}{2}\right)+a_{2}\left(\frac{h}{2}\right)^{2}+O\left(h^{3}\right)
$$

E também $P_{4}=F\left(\frac{h}{4}\right)$ da seguinte forma:

$$
F\left(\frac{h}{4}\right)=a_{0}+a_{1} \frac{h}{4}+a_{2}\left(\frac{h}{4}\right)^{2}+O\left(h^{3}\right) .
$$

Eliminando os termos de ordem superior a dois, chega-se ao seguinte sistema:

$$
\left\{\begin{array}{l}
P_{1}=a_{0}+a_{1} h+a_{2} h^{2} \\
P_{2}=a_{0}+a_{1} \frac{h}{2}+a_{2} \frac{h^{2}}{4} \\
P_{4}=a_{0}+a_{1} \frac{h}{4}+a_{2} \frac{h^{2}}{16}
\end{array}\right.
$$

Resolvendo o sistema para encontrar o valor de $a_{0}$ e fazendo $P=a_{0}$, chega-se a seguinte equação polinomial:

$$
P=\frac{8}{3} P_{4}-2 P_{2}+\frac{1}{3} P_{1}
$$




\section{Resultados}

Nesta seção é realizada uma simulação para calcular o valor de uma opção de venda americana, utilizando para isto, uma ação da Petrobras como sendo o ativo-objeto. Utiliza-se o histórico dos preços deste ativo no período de um ano (01/04/2004 a 31/03/2005), cujas informações foram obtidas no site da Petrobras, www.petrobras.com.br. O tempo para o vencimento será de 6 meses e o preço de exercício é definido no contrato. A taxa de juros considerada para a simulação será a taxa SELIC (Sistema Especial de Liquidação e Custódia) do período correspondente. Para se determinar a volatilidade é necessária uma análise dos valores passados dos ativos.

Neste trabalho, a volatilidade é obtida pela fórmula do desvio padrão, dada por:

$$
\sigma=\left(\frac{1}{n} \sum_{i=0}^{n}\left(u_{i}-\mu\right)^{2}\right)^{1 / 2}
$$

onde $\mu$ é a média dos retornos dada por:

$$
\mu=\frac{1}{n+1} \sum_{i=0}^{n} u_{i}
$$

Para esta simulação, cujo ativo objeto é a ação da Petrobras, PETR4-PN, suponha que a opção será comprada no dia 01/10/04, ou seja, na metade do tempo no qual os valores foram colhidos. Como foram observados valores em 247 dias úteis, a data de compra da opção será o dia 28/09/2004. Neste dia a ação fechou com o preço de $R \$ 93,84$. O preço de exercício será de $R \$ 100,00$. A data de vencimento será o dia 31/03/2005, ou seja, o tempo para o vencimento será de 6 meses. A taxa SELIC anual neste período era de 16,12\%, segundo informações obtidas no site do Banco Central (www.bcb.gov.br). A taxa de juros transformada para taxa de juros contínua livre de risco será:

$$
r=\ln (1+0,1612)=0,1495=14,95 \% .
$$

Para determinar a volatilidade, utilizam-se os valores encontrados nos 6 primeiros meses colhidos, ou seja, nos primeiros 122 dias úteis.

E a volatilidade histórica anual será de:

$$
\sigma_{\text {anual }}=\sqrt{250} \sigma_{\text {dia }}=0,3037 .
$$

Para esta simulação os valores iniciais são: $S_{0}=93,84, \quad T=6$ meses $=1 / 2$ ano, $\quad X=100,00, \quad r=14,95 \%$ e $\sigma=0,3037$.

Pela equação (20), o valor da opção de venda americana no dia 01/10/2004, da ação PETR4-PN é:

$$
P \cong R \$ 8,89
$$




\section{Comparações}

Nesta seção são feitas comparações, com dois métodos já existentes para calcular o valor de uma opção de venda americana, o modelo binomial e o modelo de inequações variacionais, com a finalidade de testar a qualidade do modelo baseado na extrapolação de Richardson.

Em 1979, Cox e Ross (1976) desenvolveram o modelo binomial. Este modelo considera a vida da opção dividida em pequenos intervalos de tempo de extensão $\Delta t$. Em cada intervalo de tempo, o valor do ativo pode aumentar $u$ vezes, com probabilidade $p$ ou diminuir $d$ vezes com probabilidade $1-p$, onde $u$ e $v$ estão relacionados com a variância.

As opções são avaliadas do fim ao começo da árvore. Para opções americanas é necessário verificar em cada nó da árvore, se o exercício antecipado será melhor que manter a opção por um mais um período de tempo $\Delta t$. Isto significa que para um intervalo de tempo muito pequeno, o número de análises será muito grande.

Aplicando o modelo binomial aos valores da simulação realizada, cujo ativoobjeto é a ação da Petrobras, o valor encontrado para a opção de venda americana foi:

$$
P \cong R \$ 8,86 .
$$

A tabela 1, a seguir mostra a comparação entre o resultados do método da extrapolação de Richardson e o modelo binomial para as simulação anterior.

Tabela 1

\begin{tabular}{ccc}
\hline Ativo & extrapolação & Binomial \\
\hline ação da Petrobras & $R \$ 8,89$ & $R \$ 8,86$ \\
\hline
\end{tabular}

Em 2004, Israel (2004) calculou o preço da opção de venda americana, mostrando que o sistema de inequações variacionais abaixo, ver Bensoussan e Lions (1978), possui solução única.

$$
\begin{cases}\frac{\partial U}{\partial t}+A U-r U \leq 0 \text { e } U \geq \psi, & \text { em } \mathbb{R}^{n} \times[0, T] \\ \left(\frac{\partial U}{\partial t}+A U-r U\right)(\psi-U)=0, & \text { em } \mathbb{R}^{n} \times[0, T] \\ U(X, T)=\psi(X, T), & \text { em } \mathbb{R}^{n} .\end{cases}
$$

onde, $U$ é o valor da opção de venda americana, $X$ é o preço do ativo-objeto, $t$ é o tempo para o vencimento, $r$ é a taxa de juros livre de risco e $A$ é um operador dado por:

$$
A(t)=\sum_{i, j=1}^{n} a_{i j}(X, t) \frac{\partial^{2}}{\partial X_{i} \partial X_{j}}+\sum_{j=1}^{n} b_{j}(X, t) \frac{\partial}{\partial X_{j}}
$$

onde, $a_{i j}(X, t)=\frac{1}{2} \sum_{k=1}^{n} \sigma_{i k}(X, t) \sigma_{j k}(X, t)$ e $b(X, t)$ é o vetor direção do movimento do ativo-objeto e $\sigma(X, t)$ é a matriz volatilidade. 
Nesse trabalho, o preço da opção de venda americana foi calculado utilizando o método de elementos finitos e o método de diferenças finitas no tempo para resolver a inequação variacional

$$
\frac{\partial U}{\partial t}(X, t)+\frac{1}{2} \sigma^{2} X^{2} \frac{\partial^{2}}{\partial t^{2}}(X, t)+r X \frac{\partial U}{\partial X}(X, t)-r U(X, t) \leq 0
$$

com obstáculo $\psi$ tal que $U(X, t) \geq \psi(X, t)$, onde $(X, t) \in \mathbb{R} \times \mathbb{R}^{+}$.

Calculando o valor da opção de venda americana, cujo ativo-objeto é a ação da Petrobras PETR4-PN, pelo modelo de inequação variacional, o valor encontrado para a opção de venda americana foi de:

$$
P \cong R \$ 8,87
$$

A tabela 2, a seguir mostra a comparação entre o método da extrapolação de Richardson e o método da inequação variacional para as simulação realizada.

Tabela 2

\begin{tabular}{ccc}
\hline Ativo & Extrapolação & Inequação \\
\hline ação da Petrobras & $R \$ 8,89$ & $R \$ 8,87$ \\
\hline
\end{tabular}

\section{Conclusão}

Pode-se concluir, através da simulação realizada e das comparações com os outros métodos já desenvolvidos que, apesar de não termos uma solução analítica, o modelo baseado na solução dada por Black e Scholes (1973) e na extrapolação de Richardson encontrou valores bem próximos dos valores encontrados pelos outros métodos.

A vantagem deste novo modelo em relação ao modelo binomial é justamente a discretização. O modelo binomial oferece melhores resultados conforme aumentase a discretização no tempo, o que o torna mais lento computacionalmente. Usando a extrapolação de Richardson, foi necessário dividir o intervalo de tempo em apenas quatro intervalos iguais.

Se comparado com o modelo de inequaçoes variacionais pode-se dizer que este novo modelo é mais fácil de implementar pois o resultado é obtido de uma simples equação polinomial ao invés de um sistemas de inequações variacionais, cuja resolução envolve Espaço de Sobolev com pesos, método de compacidade e regularização de domínio, ver mais detalhes em Bensoussan e Lions (1978) ou Marcozzi (2001).

De uma forma geral, as grandes contribuições do modelo baseado na solução dada por Black e Scholes (1973) e na extrapolação de Richardson são a sua simplicidade e rapidez computacional. 


\section{Referências}

Bensoussan, A. \& Lions, J. (1978). Applications Des Inéquations Variationnelles En Contrôle Stochastique. Dunod, Paris.

Black, F. \& Scholes, M. (1973). The pricing of options and corporate liabilities. Journal of Political Economy, 81:637-59.

Carr, P., Jarrow, R., \& Myneni, R. (1992). Alternative characterizations of American put options. Mathematical Finance, 2:87-106.

Cox, J. \& Ross, S. (1976). The valuation of options for alternative stochastic processes. Journal of Financial Economics, 3:145-66.

Geske, R. (1979). The valuation of compound options. Journal of Financial Economics, 7:63-81.

Israel, V. P. (2004). Inequações variacionais aplicadas ao problema do mercado de opções. Dissertação de Mestrado - IM - NCE - UFRJ.

Marcozzi, M. D. (2001). On the approximation of optimal stopping problems with application to finantial mathematics. SIAM journal on scientific computing, $22: 1865-1884$

Merton, R. C. (1973). Theory of rational option pricing. The Bell Journal of Economics and Management Science, 4:141-183. 